\title{
Counterexample to the hypothesis of functional similarity between tactile and visual pattern perception
}

\author{
JACK M. LOOMIS \\ University of California, Santa Barbara, California
}

\begin{abstract}
In earlier work, the author has demonstrated that tactile pattern perception and visual pattern perception exhibit many parallels when the effective spatial resolution of vision is reduced to that of touch, thus supporting the hypothesis that the two pattern senses are functionally similar when matched in spatial bandwidth. The present experiments demonstrate a clear counterexample to this hypothesis of functional similarity. Specifically, it was found that the lateral masking effect of a surround on tactile character recognition increases when the surround changes in composition from solid lines to dots, whereas for vision, recognition performance goes in the opposite direction. This finding necessitates some modification of the model of character recognition proposed by the author (Loomis, 1990) as it applies to the sensing of raised tactile patterns. One possible modification would be to incorporate, as the initial stage of pattern transformation, the continuum mechanics model for the skin that was developed by Phillips and Johnson (1981b).
\end{abstract}

Although the cutaneous sense and vision differ considerably in terms of biological substrate, they are both image-processing systems and thus amenable to the same type of functional description (Loomis \& Lederman, 1986). With this view, a number of experiments have compared cutaneous (tactile) pattern perception and visual pattern perception, showing that when the effective spatial bandwidths of the two modalities are closely matched, they exhibit a strong functional similarity (Loomis, 1981, 1982,1990 ). Specifically, if the visual stimuli are subjected to lowpass spatial filtering (either by the optics of the eye or by an external optical filter) so that visual recognition performance is approximately matched to that of touch, two manifestations of functional similarity emerge: (1) character sets differing in size and typography (e.g., braille, roman letters, etc.) exhibit virtually the same ordering in legibility for the two senses (Loomis, 1981,1990 ) and (2) the tactile and visual confusion matrices for a given character set are similar, both in terms of character legibility (the diagonal cell values) and in confusion errors (off-diagonal cell values) (Loomis, 1982). This congruence between the two modalities motivated the development of a process model of character recognition that is based in part on the spatial filtering idea; the model proved quite successful in predicting legibility and confusion errors for both senses (Loomis, 1990). Interestingly, the spatial filtering idea has received strong confirmation from spatial reconstructions of the recordings of two classes of mechanoreceptor units in awake

This research was supported by Grant NS15129 from NINCDS. The author thanks David Eby and Andrew Beall for assistance. Address correspondence to J. M. Loomis, Department of Psychology, University of California, Santa Barbara, CA 93106-9660. humans (Phillips, Johansson, \& Johnson, 1990)-work that follows similar studies with monkeys (Johnson \& Lamb, 1981).

Despite the overall pattern of functional similarity in that work, some differences between the two senses have always been apparent. A significant one is that, even when the two senses are matched in terms of effective spatial resolution, tactile recognition performance falls short of the essentially errorless performance attainable with vision when very large characters are used (Loomis, 1981, 1990). The author has speculated that because subjects have had much less experience with interpreting tactile patterns, they are less able to integrate the perceptual information that is available to them than in the case of vision. Other differences are more subtle. These include confusion errors that are common for one modality but not the other (Loomis, 1982) and differences in the ordering of legibility between the two senses. As an example of the latter, a comparison between characters composed of line strokes and characters of identical shape composed of dots indicated a possible interaction between character composition and modality-visual performance was just slightly better than tactile performance with dot characters and just slightly worse with line characters (Loomis, 1981, 1990).

\section{EXPERIMENT 1}

The possible interaction between sensory modality and character composition led to the present experiment. Earlier work (Loomis, 1990) had shown a very large lateral masking effect for both touch and vision-braille characters in isolation were much more legible (recognition performance $=56 \%$ for touch and $81 \%$ for vision) 
than the same braille characters surrounded by small rectangular outlines (16\% for touch and $10 \%$ for vision). This was a result predicted by the model. What would happen if the surround defined by solid lines were replaced by a surround defined by dots? Because the model predicts that the masking effect of the surround is monotonically related to its excitatory effect, reducing the number of "points" in the surround ought to decrease the masking effect. However, because a serrated line sensed by touch is perceptually more salient than a solid line of the same length, perhaps for reasons of skin mechanics (Phillips \& Johnson, 1981b), it seemed likely that the dotted surround might produce more tactile masking than a solid surround, contrary to the prediction of the model. Experiment 1 involved visual and tactile recognition of characters presented in isolation or in the presence of rectangular surrounds formed out of lines or dots.

\section{Method}

The method was essentially the same as that used in previous work (Loomis, 1981, 1982, 1990).

Stimuli. The stimuli used in the experiment are shown in Figure 1. The top panel (Set 1) depicts the 26 alphabetic characters of standard English braille (dotted braille), and the middle panel (Set 2) depicts the same characters, except the dots in adjacent rows or columns have been connected by lines to create what is referred to as connected braille. These two sets were used in constructing the other six character sets used in the experiment. The lower panel depicts all eight character sets with either the dotted braille or connected braille character for $T$ being the representative in each case. The four sets on the left used dotted braille characters as targets, either in isolation or with surrounds differing in dot density (from sparse to solid); those on the right used connected braille characters with and without surrounds.

The tactile stimuli were created by photoengraving. The eight character sets were printed by computer and then arranged in eight concentric circles in the artwork. The photoengraver then made two negatives of the artwork at slightly different magnifications, and from these made two zinc plates in which the raised patterns were

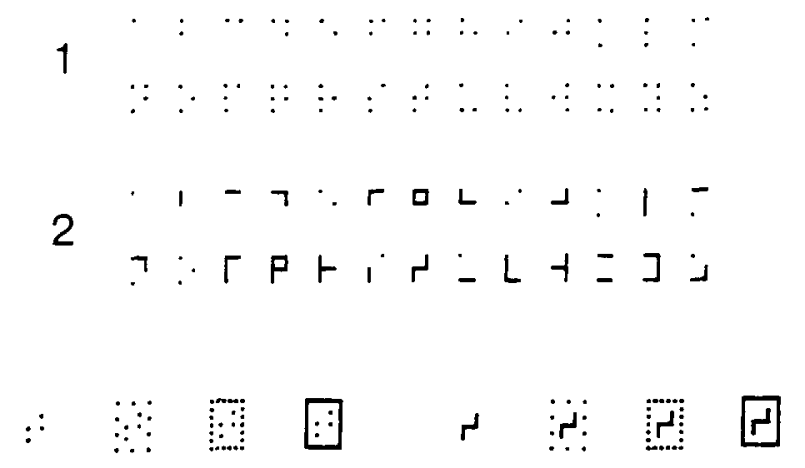

Figure 1. Stimuli used in both experiments. Character Set 1 consisted of the alphabetic characters of standard English braille (dotted braille). Character Set 2 was the same, except that lines connected dots in adjacent rows or columns (connected braille). The bottom panel represents the eight character sets used in the experiments. On the left are the four sets using dotted braille with and without surrounds, with the character $T$ shown in each case. On the right are the four sets using connected braille, again with character $T$. exact facsimiles of the transparent figures in the negatives. The scales of the two plates (and negatives) were such that the horizontal and vertical center-point spacings of the braille cell were 3.0 and $2.5 \mathrm{~mm}$, respectively, for the larger plate; for the smaller plate these values were $91 \%$ as large. From a sample of caliper measurements, the raised patterns averaged 0.64 and $0.53 \mathrm{~mm}$ in height for the larger and smaller plates, respectively; these heights are more than adequate for good tactile sensing (Loomis, 1985). The difference in heights for the two plates was of no consequence, because earlier work had shown essentially constant performance for this range of height variation (Loomis, 1985).

For stimulus presentation, either plate could be rotated about its center while horizontally supported by three equally spaced supports, one of which was a force transducer (Sensotec Model 34). After calibration, the signal from the transducer allowed the IBM PC computer to record the average incremental force obtained during touching on each trial. By rotation of the plate, any desired character within a set could be positioned beneath a subject's right index finger, which was held in position by an adjustable finger guide. The subject's arm and hand rested on supports. Different character sets were selected by positioning the arm, hand, and finger guide at different radii from the plate's center.

The visual stimuli, prior to optical lowpass spatial filtering, were the transparent characters in the negatives used by the photoengraver to make the plates. Each negative was affixed to a Plexiglas disk and mounted vertically. On one side of the disk, a tungsten source, condensing lens, and shutter provided timed and collimated illumination of a restricted portion of the disk, with rotation and lateral displacement of the disk permitting back-illumination of any desired character. On the other side of the disk were two parallel diffusing sheets, mounted vertically. With back parallel projection of any character onto the first of the two diffusers, the desired lowpass spatial filtering could be accomplished by adjusting the separation between the two diffusers. Earlier work (Loomis, 1981; see also Loomis \& Lederman, 1986) had determined the setting of the optical filter that resulted in a match between visual and tactile resolution performance. That setting was again used here; from earlier measurements, this filter had a Gaussian point spread function (measured in the plane of the second diffuser), with a full width at halfamplitude of $5.8 \mathrm{~mm}$.

Photometric measurement indicated that within the plane of the second diffuser (the output plane of the filter), the spread function corresponding to a single point input had a luminous irtensity of $5.0 \times 10^{-6} \mathrm{~cd}$. With the viewing distance of $81 \mathrm{~cm}$, such a stimulus was well within the photopic range (approximately $1.5 \mathrm{log}$ units above foveal cone threshold). Each millimeter in the plane of the second diffuser subtended $4.24^{\prime}$ of arc.

Subjects. Five women and 3 men, all in their twenties, participated as paid observers. All had at least $20 / 30$ vision, uncorrected or corrected, as assessed by Keystone Orthoscope. Some of them had participated in similar experiments, but none were aware of the purpose of the experiment.

Procedure. Each subject participated in six 2-h sessions. Within each session, the subject experienced all 32 conditions ( 8 sets $\times$ 2 sizes $\times 2$ modalities). The conditions were blocked by modality and within each modality by stimulus size; for a given size and modality, the order of the eight character sets was randomized. During the first session, half of the subjects began with vision and half with touch. Thereafter, the starting modality alternated from session to session for each subject. For each of the 32 conditions, five characters were randomly selected without replacement as stimuli, giving a total of 160 trials per session per subject. The subjects received feedback on each trial.

In the tactile task, all the stimuli were presented to the approximate center of the distal pad of the right index finger. The sides of the finger guide were spaced far enough apart that the finger could make slight motions over the surface of the character, with some slippage between corneum and pattern permitted. Contact with 
the character was limited to $2 \mathrm{sec}$. The subjects were instructed to make sure that the fingerpad always made even contact with the entire character. Average force in grams was measured for the duration of tactile scanning.

In the visual task, the subject viewed each stimulus with the left eye; the right eye was occluded with an eye patch. Stimulus duration was $2 \mathrm{sec}$. The room was dark, and no light appeared before or after the stimulus presentation.

As in previous work, the subjects were taught alphabetic labels for the braille characters in a session prior to beginning the experiment proper; the same labels were used for corresponding dotted and connected braille. Learning was accomplished with normal viewing of a computer-driven CRT. A subject first went through the 26 alphabetic characters of conventional braille in random order as many times as needed to achieve five errorless runs through the set. Then the connected braille characters were tested until three errorless runs were attained. On subsequent days of the experiment proper, the subjects had to demonstrate that they still knew the labels of the 26 braille characters.

\section{Results and Discussion}

Recognition performance measured in percent correct is given in Figure 2, as a function of sensory modality, stimulus size, target type (dotted vs. connected braille), and mask type (none, sparse, medium, and solid). The
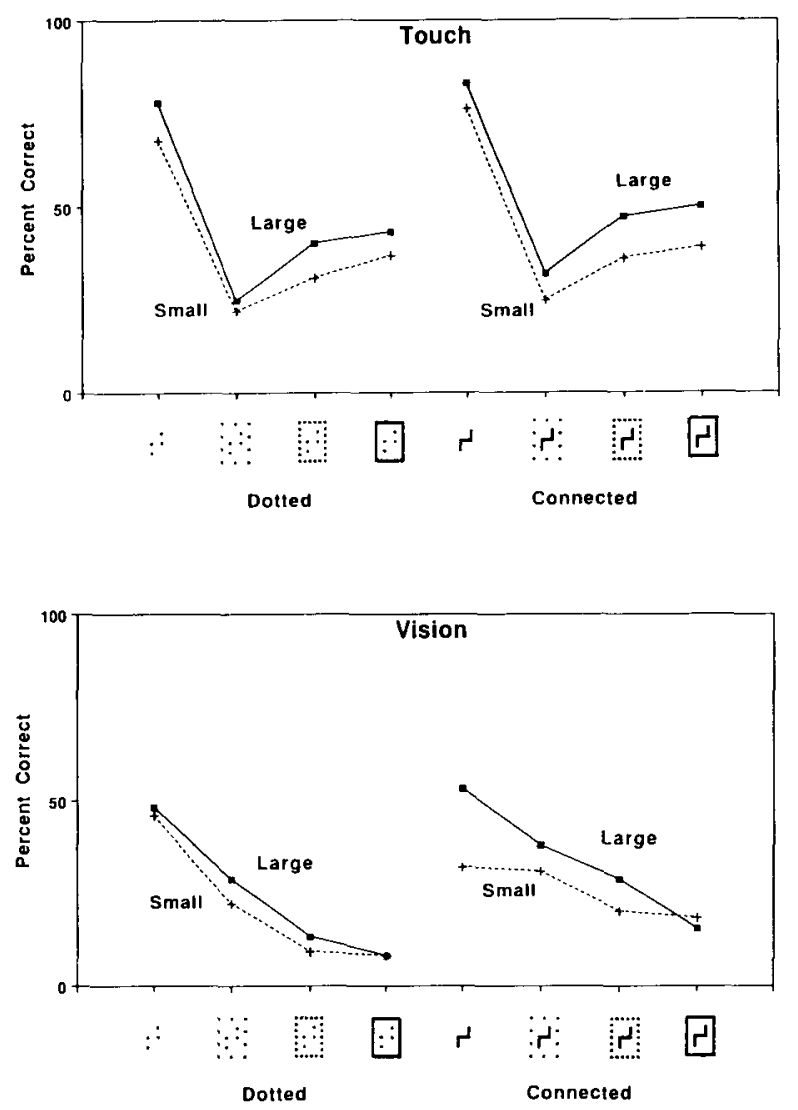

Figure 2. The results of Experiment 1. Recognition performance (percent correct) is given as a function of target type (dotted and connected braille), mask type (including no mask), character size, and modality.
Table 1

Results of Analysis of Variance for Experiment 1

\begin{tabular}{lccrc}
\hline \multicolumn{1}{c}{ Test } & $d f 1$ & $d f 2$ & \multicolumn{1}{c}{$F$} & \multicolumn{1}{c}{$p$} \\
\hline Modality & 1 & 7 & 27.52 & .0012 \\
Size & 1 & 7 & 43.59 & .0003 \\
Target & 1 & 7 & 4.66 & n.s. \\
Mask & 3 & 5 & 140.14 & $<.0001$ \\
Modality $\times$ size & 1 & 7 & 1.50 & n.s. \\
Modality $\times$ target & 1 & 7 & 0.12 & n.s. \\
Modality $\times$ mask & 3 & 5 & 10.19 & .0143 \\
Size $\times$ target & 1 & 7 & 6.30 & .0404 \\
Size $\times$ mask & 3 & 5 & 4.95 & n.s. \\
Target $\times$ mask & 3 & 5 & 2.61 & n.s. \\
Modality $\times$ size $\times$ target & 1 & 7 & 1.15 & n.s. \\
Modality $\times$ target $\times$ mask & 3 & 5 & 24.35 & .0021 \\
Size $\times$ target $\times$ mask & 3 & 5 & 1.61 & n.s. \\
Modality $\times$ size $\times$ target $\times$ mask & 3 & 5 & 8.29 & .0029 \\
\hline
\end{tabular}

Note-n.s. $=$ not significant.

results of the four-way repeated measures analysis of variance (ANOVA) are given in Table 1. The most important and statistically significant effects are discussed below.

The significant main effect of size replicated earlier results (Loomis, 1981, 1985) and was thus fully expected; the effect is as small as it is because the increase in size from one condition to the other was only $10 \%$. The manipulation of size was included to assess the generality of the effects of the other variables. The fact that it interacted only slightly with target and with the other variables in the four-way term shows that the effects of manipulating the other variables largely generalize across the small range of the size manipulation. The theoretical significance of the effect of size has been discussed at length elsewhere (Loomis \& Lederman, 1986).

The effect of modality was opposite to that found in earlier experiments (Loomis, 1981, 1990). Here, tactile performance proved superior, whereas visual performance has been superior in the past. There is no great import in one modality performing better than the other, because the relative performance of the two can be readily modified by an adjustment of the optical lowpass filter used in the visual task. However, why the ordering in this experiment differed from that of the earlier studies is puzzling and is considered in Experiment 2.

Of greater interest is the significant main effect of mask condition. As can be seen in Figure 2, all conditions in which a surround is present produced lower performance than when no surround is present; this indicates that all surrounds, regardless of their type, exert both tactile and visual lateral masking effects-not an unexpected result (see, e.g., Loomis, 1978; Loomis \& Apkarian-Stielau, 1976; Weisenberger \& Craig, 1982).

The result of greatest theoretical import was the significant interaction between type of surround and sensory modality. For vision, as the surround changed from sparse dots to solid lines, recognition performance declined, indicating that the amount of lateral masking increases with number of points defining the surround. In contrast, for touch, the smoother the lines defining the surrounds, the 
better the performance, indicating that masking is greatest with sparse surrounds. This important result will be discussed in the General Discussion.

The significant three-way interaction between modality, target, and mask can easily be noted in Figure 2 . Although the two functions on the left of the upper panel (touch) are essentially the same as those on the right in terms of both performance level and shape, the corresponding functions in the lower panel (vision) are quite different; those for normal braille on the left are lower and steeper than those for the line braille on the right.

Of secondary interest are the average force readings made while the subjects were touching the characters. For the eight character sets, reading from left to right in the bottom panel of Figure 1, the mean force values were 61, $81,75,68,68,78,69$, and $68 \mathrm{~g}$ for the large plate, and $58,77,74,70,60,69,70$, and $74 \mathrm{~g}$ for the small plate. The product-moment correlations between these values and the respective percent-correct scores were -.81 for the large plate and -.90 for the small plate, indicating a relationship between performance and contact force. Because the subjects presumably chose the contact forces that were found to be most effective in sensing the patterns, it seems likely that the largest forces observed in
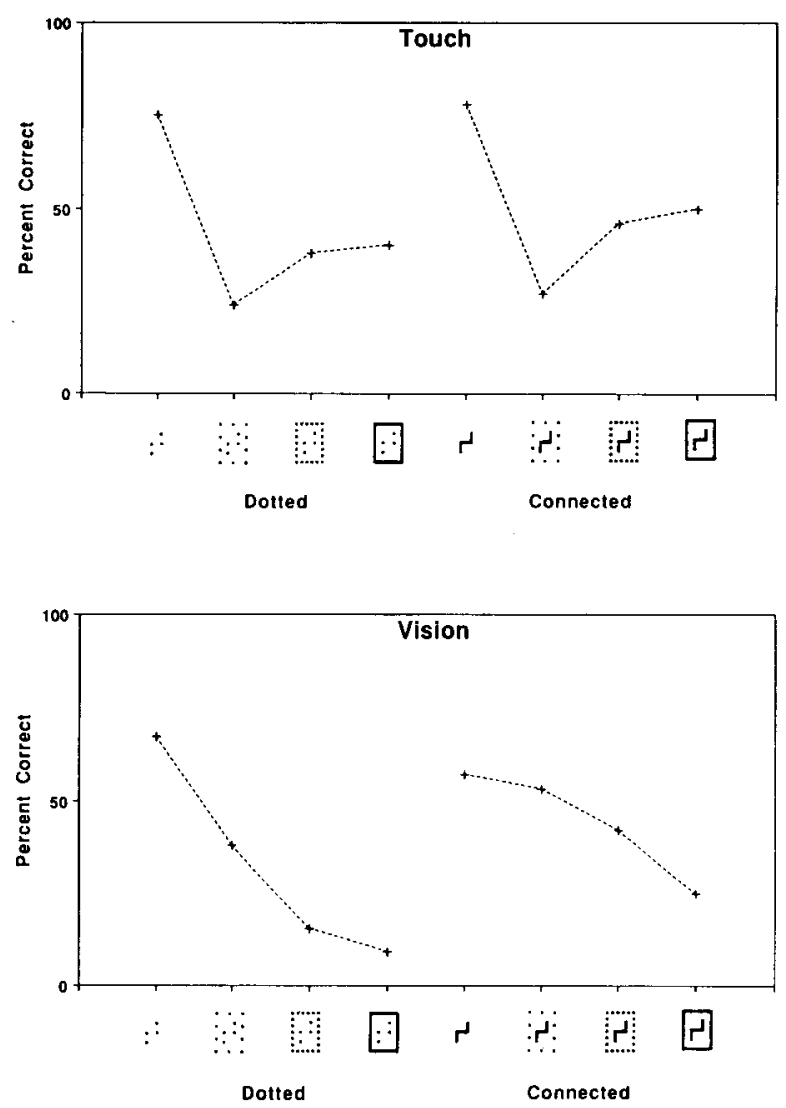

Figure 3. The results of Experiment 2. Recognition performance (percent correct) is given as a function of target type (dotted and connected braille), mask type (including no mask), and modality.
Table 2

Results of Analysis of Variance for Experiment 2

\begin{tabular}{lccrc}
\hline \multicolumn{1}{c}{ Test } & $d f 1$ & $d f 2$ & \multicolumn{1}{c}{$F$} & $p$ \\
\hline Modality & 1 & 4 & 8.01 & .0472 \\
Target & 1 & 4 & 11.18 & .0287 \\
Mask & 3 & 2 & 348.81 & .0029 \\
Modality $\times$ target & 1 & 4 & 5.24 & n.s. \\
Modality $\times$ mask & 3 & 2 & 53.76 & .0183 \\
Target $\times$ mask & 3 & 2 & 12.50 & n.s. \\
Modality $\times$ target $\times$ mask & 3 & 2 & 37.39 & .0262 \\
\hline
\end{tabular}

Note - n.s. $=$ not significant.

the sparse surround conditions (in which performance was worst) were the result of the subjects' applying more force in an attempt to enhance performance rather than the other way around-that the large forces per se were causing the poor performance.

\section{EXPERIMENT 2}

Experiment 2 was prompted by the fact that visual performance proved worse than tactile performance in Experiment 1 , contrary to what had been found in earlier research. Because the vision apparatus in Experiment 1 had been slightly modified from that used in the earlier experiments, it was noted after the experiment that the modification was producing a fair amount of reflected light within the optical system, which seemed to be acting as a veil, thus reducing the contrast of the visual patterns. In Experiment 2, care was taken to minimize this unwanted reflection.

\section{Method}

Stimuli, Apparatus, and Procedure. Besides the reduction of reflected light within the optical path, the other minor changes in method are indicated as follows. (1) No force measurements were taken in the touch experiment. (2) Only the larger character set of Experiment 1 was employed here, both for touch and vision. (3) Within each of the six experimental sessions of the experiment proper, each subject received 10 randomly selected characters from each of the eight character sets (large size only), for both touch and vision.

Subjects. Three women and 2 men, ranging in age from 25 to 31 years, participated as paid observers. All had at least 20/30 vision, uncorrected or corrected, as assessed by Keystone Orthoscope. Some of them had participated in similar experiments, but none were aware of the purpose of the experiment.

\section{Results and Discussion}

Recognition performance measured in percent correct is given in Figure 3, as a function of sensory modality, target type, and mask type. The results of the three-way repeated measures ANOVA are given in Table 2.

For the most part, the results replicated those of Experiment 1 . To begin with, there was a significant effect of modality, indicating that, on the average, touch still gave slightly better performance than vision, in contrast to what had been found in earlier work (Loomis, 1981, 1990). Perhaps because of our efforts to reduce the reflected light within the optical system, visual perfor- 
mance was considerably better than in Experiment 1, but the advantage shown by touch still remained. Why the better overall performance of vision obtained in earlier work was not obtained here remains a mystery, but, as stated earlier, there is little import in this performance difference.

There was a significant effect of target type at the .05 level, probably reflecting the higher level of performance of connected braille relative to conventional braille for vision. More importantly, the highly significant effect of mask confirmed what is by now obvious - that the dotted and solid surrounds did interfere with recognition of the targets.

As before, the most important result is the highly significant interaction between modality and mask type. For touch, dotted surrounds had the most deleterious effect for touch, whereas for vision, solid surrounds had the most deleterious effect.

Finally, as in Experiment 1, the three-way interaction between target, mask, and modality reflects the differences in performance level and shape of the two functions in the bottom panel (vision) that do not appear in the upper panel (touch).

\section{GENERAL DISCUSSION}

In both experiments, a prominent interaction between modality and mask type was observed. As can readily be seen in Figures 2 and 3, changing from sparse to solid surrounds caused tactile performance to increase and visual performance to decrease, indicating that the masking effect of a sparse surround is greatest for touch and least for vision. This difference in performance constitutes a clear counterexample to the hypothesis of functional similarity between tactile and visual pattern perception asserted by the author (Loomis, 1981, 1982, 1990; Loomis \& Lederman, 1986).

Given this functional difference between the two senses, it is clear that the model of character recognition and legibility developed by the author (Loomis, 1990) cannot apply equally well to both senses. For which modality is the model more applicable and how might it be modified to better fit the other?

Figure 4 shows the model predictions for the character sets used in Experiment 2. These predictions were obtained by using a minor modification of the basic model (reported in Loomis 1990, p. 117), which allowed a better fit for the character sets with surrounds (Sets 22 and 23 in that work). As is evident in Figure 4, the four performance functions are similar in shape and relative vertical position to those in Figure 2, obtained for vision in Experiment 1. The primary difference is that the model predicts substantially higher performance than was obtained; recall, however, that changes in the apparatus did result in visual performance for Experiment 2 that was much closer to that predicted by the model.

The fact that the model was quite successful in predicting the observed effects of manipulating mask type, target type, and character size on visual performance lends

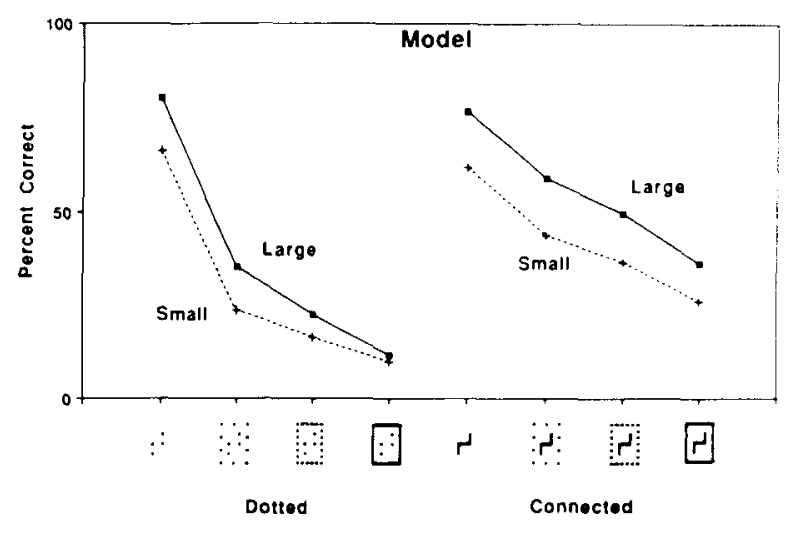

Figure 4. The results of the simulations using Loomis's (1990) model. Predicted recognition performance (percent correct) is given as a function of target type (dotted and connected braille), mask type (including no mask), and character size.

further support to its applicability to visual character recognition beyond that adduced in Loomis (1990). As discussed there (p. 117), the surrounds exert their masking effects in two ways: (1) by the compressive nonlinearity assumed to intervene between the linear spatial filtering stage and the computation of some distance metric in the template matching stage, and (2) by the rescaling of the filtered patterns by the overall intensity within the target region. Because the sparse surrounds consist of far fewer points than the continuous surrounds, they cause less rescaling and nonlinear compression of the pattern representations than do the solid surrounds. This means that differences computed between targets in the template matching stage are greater for sparse surrounds than for line surrounds, implying greater discriminability with the sparse surrounds. Another result predicted by the model is the clear target $\times$ mask interaction for vision (the different shapes of the performance curves for the left and right panels of Figure 2). Because the connected braille targets have more "pixels" than the dotted braille targets, they should be more resistant to the masking effects of the encroaching surround under conditions of blurring (lowpass spatial filtering).

Because the tactile and visual performance functions differ greatly in Figures 2 and 3, the relative success of the model for vision amounts to a relative failure for touch. How might the model be modified to account for the fact that dotted surrounds exert greater masking effects for touch than do line surrounds? As mentioned in the introduction, serrating a solid raised line seems to make it more tangible. A likely explanation is provided by the work of Johnson and Phillips (1981; Phillips \& Johnson, 1981a, 1981b). Recordings from mechanoreceptor first-order afferents in the monkey revealed that when a rectangular plate is impressed upon some portion of the skin, the greatest neural activity is produced near the edges of the disk. Phillips and Johnson showed that this "edge enhancement" is a consequence of continuum skin mechanics. The downward forces acting to produce a rect- 
angular deformation of the skin are unevenly distributed with the greatest forces acting at the edges of the plate; if gaps are introduced into the plate, large forces act at their edges as well. By similar reasoning, one can see that a solid line (such as those of the solid surround) would exert lower forces along its internal portion than a serrated line of the same end-to-end length. Evidence of reduced responding to internal segments of lines and internal components of two-dimensional patterns (e.g., braille and letters) has been reported by Johnson and his colleagues (Johnson \& Lamb, 1981; Vega-Bermudez, Johnson, \& Hsiao, 1991). Thus, it could well be that incorporating an initial stage representing continuum skin mechanics into the model of character recognition described above might predict the increase in masking when going from a solid to a sparse surround. Moreover, it might lead to an improvement in the model, even for character sets without surrounds (see Vega-Bermudez et al., 1991, for some promising results in this direction).

It should be noted that the above discussion assumes that the touch patterns are raised stimuli. If the tactile patterns are produced by either a vibrotactile or electrotactile display instead, the results might be more in line with those of vision. Whereas the perception of raised patterns probably reflects the property of the skin mechanics discussed above, it seems likely that a solid surround presented on a vibrotactile or electrotactile display will produce much greater mechanoreceptor excitation than a sparse surround, with consequently greater masking of a target. With this in mind, the author conducted an experiment similar to Experiments 1 and 2, using the Optacon display instead of raised patterns. Unfortunately, recognition performance levels in the masking conditions were so low (around 10\%) that no conclusion could be drawn as to whether the results were more like those of vision or those obtained with raised patterns. This much lower performance obtained with the Optacon patterns, despite their being larger than the raised patterns of Experiments 1 and 2 , has been noted previously (Loomis \& Lederman, 1986) and reflects the fact that the Optacon stimulates only FA I mechanoreceptors (Santoro, Palmer, \& Gardener, 1987) rather than the more acute SA I mechanoreceptors stimulated by raised patterns (Johnson \& Lamb, 1981; Phillips et al., 1990; VegaBermudez et al., 1991).

In summary, the present experiments demonstrate a clear counterexample to the hypothesis of functional similarity between tactile and visual pattern perception. As such, it requires some modification of the basic model of character recognition proposed by Loomis (1990) as it applies to the sensing of raised tactile patterns. However, showing that the model fails for these particular conditions is not a reason for wholesale rejection of the model for touch, because the model is otherwise quite successful. What it means is that a modification of the model, perhaps along the lines of the continuum mechanics model of Phillips and Johnson (1981b), might lead to considerable improvement in its predictions for character sets with and without surrounds.

\section{REFERENCES}

Johnson, K. O., \& LAMB, G. D. (1981). Neural mechanisms of spatial tactile discrimination: Neural patterns evoked by braille-like dot patterns in the monkey. Journal of Physiology, 310, 117-144.

Johnson, K. O., \& Phillips, J. R. (1981). Tactile spatial resolution: 1. Two-point discrimination, gap detection, grating resolution, and letter recognition. Journal of Neurophysiology, 46, 1177-1191.

Loomis, J. M. (1978). Lateral masking in foveal and eccentric vision. Vision Research, 18, 335-338.

Loomis, J. M. (1981). On the tangibility of letters and braille. Perception \& Psychophysics, 29, 37-46.

Loomis, J. M. (1982). Analysis of tactile and visual confusion matrices. Perception \& Psychophysics, 31, 41-52.

Loomis, J. M. (1985). Tactile recognition of raised characters: A parametric study. Bulletin of the Psychonomic Society, 23, 18-20.

Loomis, J. M. (1990). A model of character recognition and legibility. Journal of Experimental Psychology: Human Perception \& Performance, 16, 106-120.

LoOmis, J. M., APKarian-Stielau, P. (1976). A lateral masking effect in tactile and blurred visual letter recognition. Perception \& Psychophysics, 20, 221-226.

LoOMIs, J. M., \& LedermaN, S. J. (1986). Tactual perception. In K. R. Boff, L. Kaufman, \& J. P. Thomas (Eds.), Handbook of perception and human performance: Vol. 2: Cognitive processes and performance (pp. 31-1-31-41). New York: Wiley.

Phillips, J. R., Johansson, R. S., \& Johnson, K. O. (1990). Representation of braille characters in human nerve fibres. Experimental Brain Research, 81, 589-592.

PhILlips, J. R., \& Johnson, K. O. (1981a). Tactile spatial resolution: 2. Neural representation of bars, edges, and gratings in monkey primary afferents. Journal of Neurophysiology, 46, 1192-1203.

PhilliPs, J. R., \& JoHNson, K. O. (1981b). Tactile spatial resolution: 3. A continuum mechanics model of skin predicting mechanoreceptor responses to bars, edges, and gratings. Joumal of Neurophysiology, 46, $1204-1225$.

Santoro, J. A., Palmer, C. I., \& Gardner, E. P. (1987). Responses of mechanoreceptors to stripe patterns on the Optacon. Society of Neurosciences Abstracts, 13, 472.

Vega-Bermudez, F., Johnson, K. O., \& Hsiao, S. S. (1991). Human tactile pattern recognition: Active versus passive touch, velocity effects, and patterns of confusion. Journal of Neurophysiology, 65, 531-546.

Weisenberger, J. M., \& Craig, J. C. (1982). A tactile metacontrast effect. Perception \& Psychophysics, 31, 530-536.

(Manuscript received September 25, 1992; revision accepted for publication January 13, 1993.) 